\title{
Evolution of a chronic dissecting aneurysm on magnetic resonance imaging in a pediatric patient
}

\author{
Long Chen, MD, PhD, ${ }^{1,2}$ Ivanna Yau, MN, NP-Peds, ${ }^{3}$ Gabrielle deVeber, MD, ${ }^{3}$ Peter Dirks, MD, PhD, ${ }^{4}$ \\ Derek Armstrong, MD, ${ }^{5}$ and Timo Krings, MD, $\mathrm{PhD}^{1,6}$
}

\begin{abstract}
'Division of Neuroradiology, Department of Medical Imaging, Toronto Western Hospital, University Health Network, Toronto; Departments of ${ }^{3}$ Neurology, ${ }^{4}$ Neurosurgery, and ${ }^{5}$ Radiology, Hospital for Sick Children, Toronto; ${ }^{6}$ Department of Neurosurgery, University of Toronto, Ontario, Canada; and 'Department of Interventional Radiology, The First Affiliated Hospital of Soochow University, Suzhou, Jiangsu, China
\end{abstract}

\begin{abstract}
Clinical and imaging manifestations of the so-called partially thrombosed aneurysm (PTA) are different from those of the classic intracranial saccular aneurysm. Given some of their peculiar imaging features, it had been hypothesized that some PTAs occur due to repeated intramural hemorrhages. The authors present a case of PTA that evolved from an acute dissecting aneurysm as shown by serial imaging. A previously healthy 5-year-old boy had a sudden onset of left hemiparesis. Initial MRI sequences showed a perforating vessel infarction in the right basal ganglia area secondary to an acute distal middle cerebral artery (MCA) dissection as demonstrated on conventional angiography. Conservative management with close observation of this dissection was chosen, and serial MRI studies revealed layering of blood of various ages within the wall of an aneurysmal outpouching of the MCA, thereby leading to the imaging appearance of a PTA. The findings in this case indicate that some PTAs may be caused by repeated or chronic dissections, with blood entering the wall through an endothelial defect. Understanding the pathological mechanism underlying the formation of these aneurysms will help inform appropriate treatment strategies.
\end{abstract}

http://thejns.org/doi/abs/10.3171/2014.10.PEDS14221

KEY WORDS partially thrombosed aneurysm; dissecting aneurysm; magnetic resonance image; vascular disorders

$\mathrm{T}$ He classic definition of an aneurysm as a focal outpouching of the arterial vessel wall neglects the different causes that underlie the formation of these lesions, and fails to provide treatment strategies tailored toward the underlying pathological mechanism. Thus, a classification of aneurysms based purely on size and location (such as that provided by the International Study of Unruptured Intracranial Aneurysms [ISUIA] or the International Subarachnoid Aneurysm Trial [ISAT]) only makes sense if the same type of aneurysm (i.e., only saccular aneurysms) is evaluated, keeping in mind that other types of aneurysms (dissecting, mycotic, traumatic, or tumorous) will have different natural histories and, likewise, may require different treatment strategies. ${ }^{11,18}$

One peculiar aneurysm subtype is the so-called partially thrombosed aneurysm (PTA), the clinical and imaging manifestations of which are different from those of a classic saccular aneurysm. ${ }^{6}$ In the late 1970s, it was Schubiger and colleagues who speculated that these "aneurysms" are caused by a disease process of the vessel wall rather than by intraluminal factors that resulted from repeated intramural hemorrhages..$^{14}$ Multiple studies subsequently confirmed this speculation by using diagnostic imaging techniques, direct surgical inspection, or histopathological investigation. ${ }^{1,5,12,13,16,17,19}$ It was suggested that certain PTAs arise from repeated hemorrhages of the vasa vasorum within the vessel wall, whereas other PTAs occur in relation to a chronic dissecting process with repeated intramural hemorrhages stemming from an incompletely healed acute dissection with intimal tearing. However, there has not yet been any serial imaging evidence to support the latter pathomechanism; i.e., the evolution of an acute dissecting aneurysm into a PTA, thereby rendering this pathomechanism hypothetical. We report the gradual evolution process from an acute intracranial dissecting aneurysm to a PTA, as witnessed on repeated MRI.

ABBREVIATIONS MCA = middle cerebral artery; MRA = magnetic resonance angiography; PTA = partially thrombosed aneurysm; TOF = time of flight. SUBMITTED April 28, 2014. ACCEPTED October 21, 2014.

INCLUDE WHEN CITING Published online December 5, 2014; DOI: 10.3171/2014.10.PEDS14221.

DISCLOSURE The authors report no conflict of interest concerning the materials or methods used in this study or the findings specified in this paper. 


\section{Case Report}

History and Examination

A previously healthy 5-year-old boy presented with a sudden onset of left hemiparesis. Initial MRI sequences showed an acute perforating vessel infarction of the right basal ganglia within the territory of the lateral lenticulostriate arteries. A time-of-flight (TOF) MR angiography (MRA) study demonstrated a right-sided dumbbell-shaped middle cerebral artery (MCA) aneurysm just proximal to the MCA bifurcation, with preaneurysmal MCA narrowing. The vessel wall surrounding the aneurysm demonstrated a crescent-shaped T1 hyperintense rim indicating intramural hematoma. The patient underwent cerebral angiography on the same day, which revealed focal preaneurysmal narrowing and an irregularly shaped aneurysm proximal to the normal MCA bifurcation, with delayed filling and emptying of the more cranial portion of the aneurysm (Fig. 1). Imaging findings, together with the clinical presentation of stroke, age group, and the location and shape of the aneurysm and the preaneurysmal arterial segment, led us to believe that the patient had an acute dissection of the proximal MCA. We speculated that this had been caused by intramural hematoma shearing, subsequent occlusion of the lateral lenticulostriate perforator, and the weakening of the wall, leading to aneurysmal dilation.
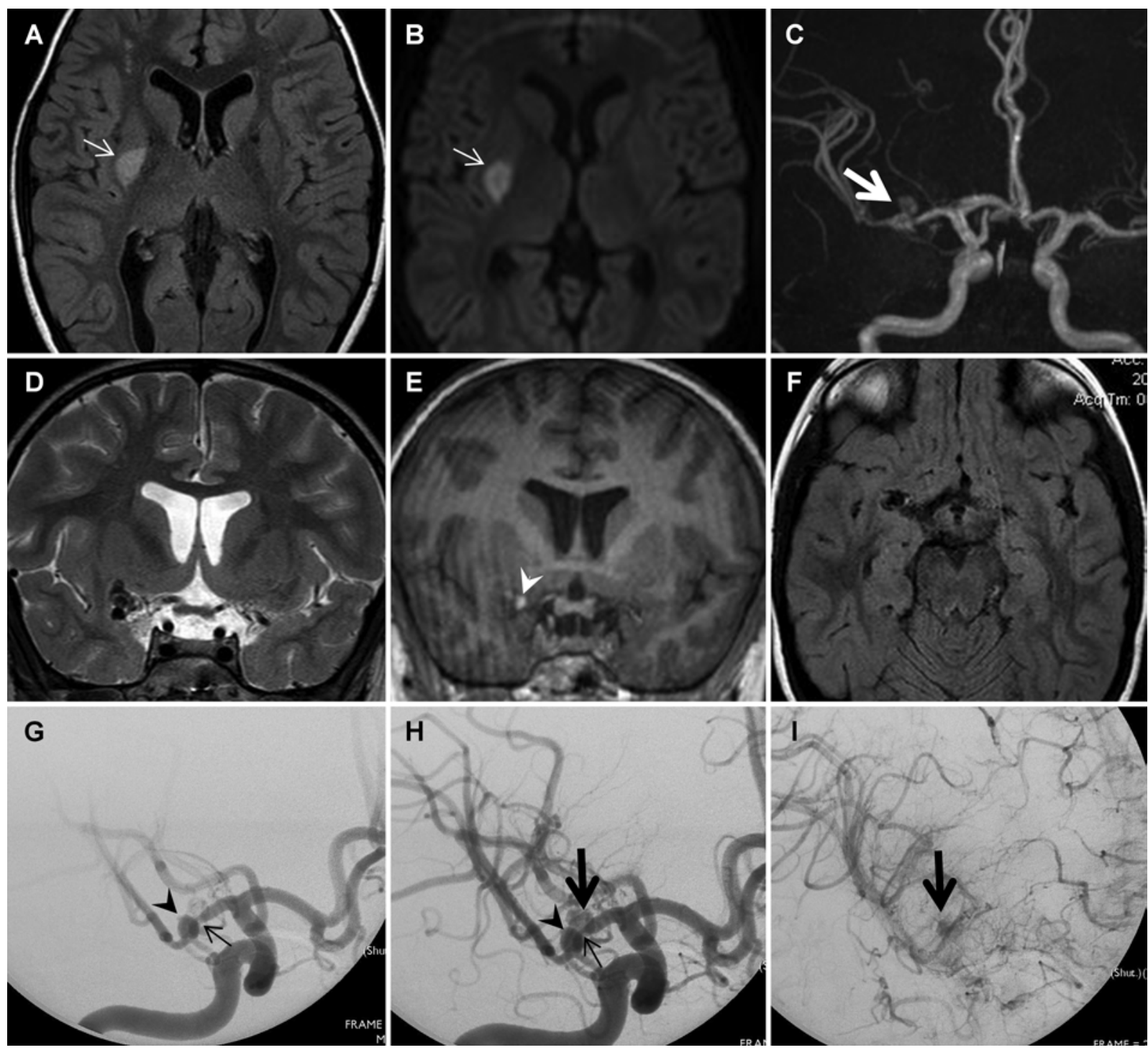

FIG. 1. Initial MRI sequences obtained at presentation. A: Axial T2 FLAIR image. B: Axial diffusion-weighted image. C: A TOF MRA study. D: Coronal T2-weighted image. E: Coronal T1-weighted image. F: Axial T2 FLAIR image. G-I: Right internal carotid digital subtraction angiograms. The MRI studies show a perforating vessel infarction in the right basal ganglia area (arrow in panels $\mathrm{A}$ and $\mathrm{B}$ ). The TOF MRA demonstrates a right-sided dumbbell-shaped MCA aneurysm (arrow in panel C) just proximal to the MCA bifurcation, with preaneurysmal MCA narrowing. The vessel wall surrounding the aneurysm demonstrates a crescentshaped $\mathrm{T} 1$ hyperintense rim indicating intramural hematoma (arrowhead in panel $\mathrm{E}$ ). The angiogram shows focal preaneurysmal narrowing (thin arrow in panels $\mathrm{G}$ and $\mathrm{H}$ ), an irregularly shaped aneurysm (arrowhead in panels $\mathrm{G}$ and $\mathrm{H}$ ) proximal to the normal MCA bifurcation, and delayed filling and emptying of the more cranial portion of the aneurysm (thick arrow in panels $\mathrm{H}$ and I). All of these images suggest a dissecting aneurysm of the right MCA. 


\section{Monitoring}

Given the lack of subarachnoid hemorrhage and the perceived risk of endovascular or surgical treatment of this lesion, we opted to monitor the patient closely with serial MRIs that were performed on the 12th, 35th, and 60th day from clinical onset. Clinically, the patient improved significantly during this period; he had only a mild residual hand weakness on Day 60. Serial MRIs demonstrated stability in the size of the perfused part of the aneurysm as indi- cated by the T2 flow void and the TOF MRA. However, the wall of the aneurysm demonstrated a gradual increase in size and a change in the imaging characteristics, with crescent-shaped areas of T1 hypersignal indicating methemoglobin apposition in an onion skin-like fashion (Fig. 2). Of interest, the new hemorrhages appeared distant from the perfused lumen of the aneurysm at the cranial wall of the lesion (Fig. 3). There was a mild interval increase in perianeurysmal edema, presumably related to mass effect.
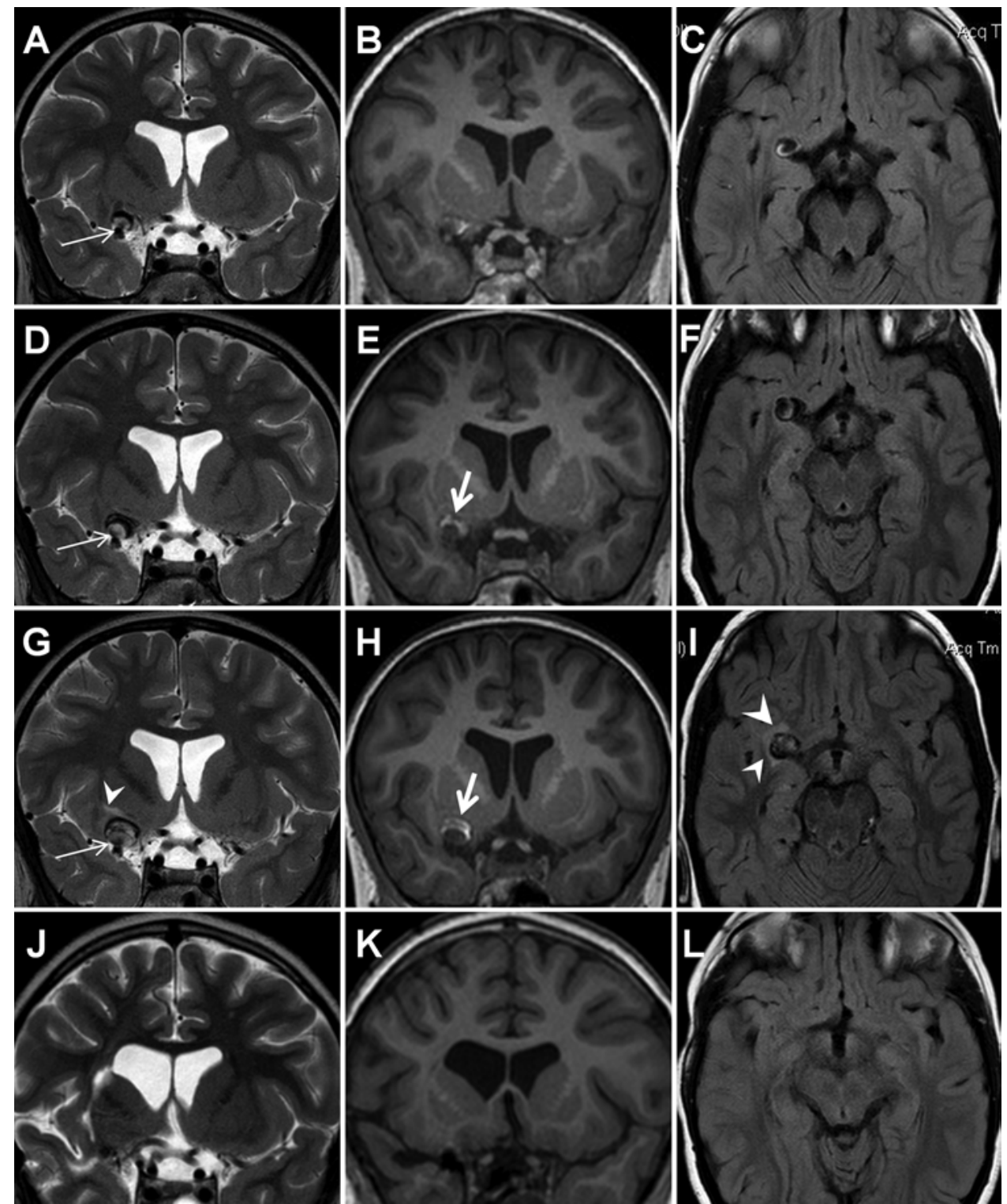

FIG. 2. Follow-up MRI sequences showing interval changes during the 2 months after initial images were obtained. The first row shows the serial images obtained 12 days after initial MRI; the second row shows the images obtained 35 days after initial MRI; and the third row shows the images obtained 60 days after initial MRI. The fourth row shows the images obtained 1 year after surgery. A, D, G, and J: Coronal T2-weighted images. B, E, H, and K: Coronal T1-weighted images. C, F, I, and L: Axial T2 FLAIR images. The MRI sequences demonstrate stable size of the perfused part of the aneurysm as indicated by the T2 flow void (thin arrow in panels A, D, and G). However, the wall of the aneurysm demonstrates a gradual increase in size and a change in the imaging characteristics, with crescent-shaped areas of T1 hypersignal indicating methemoglobin apposition in an onion skin-like fashion (thick arrow in panels E and H). The T2 and T2 FLAIR sequence images show cerebral parenchyma high signal around the aneurysm at the late stage of follow-up (arrowheads in panels $\mathrm{G}$ and I), indicating the existence of perifocal edema presumably related to increased mass effect. Follow-up after 1 year demonstrates complete obliteration of the aneurysm, regression of the perifocal edema, and no evidence of new ischemia. 

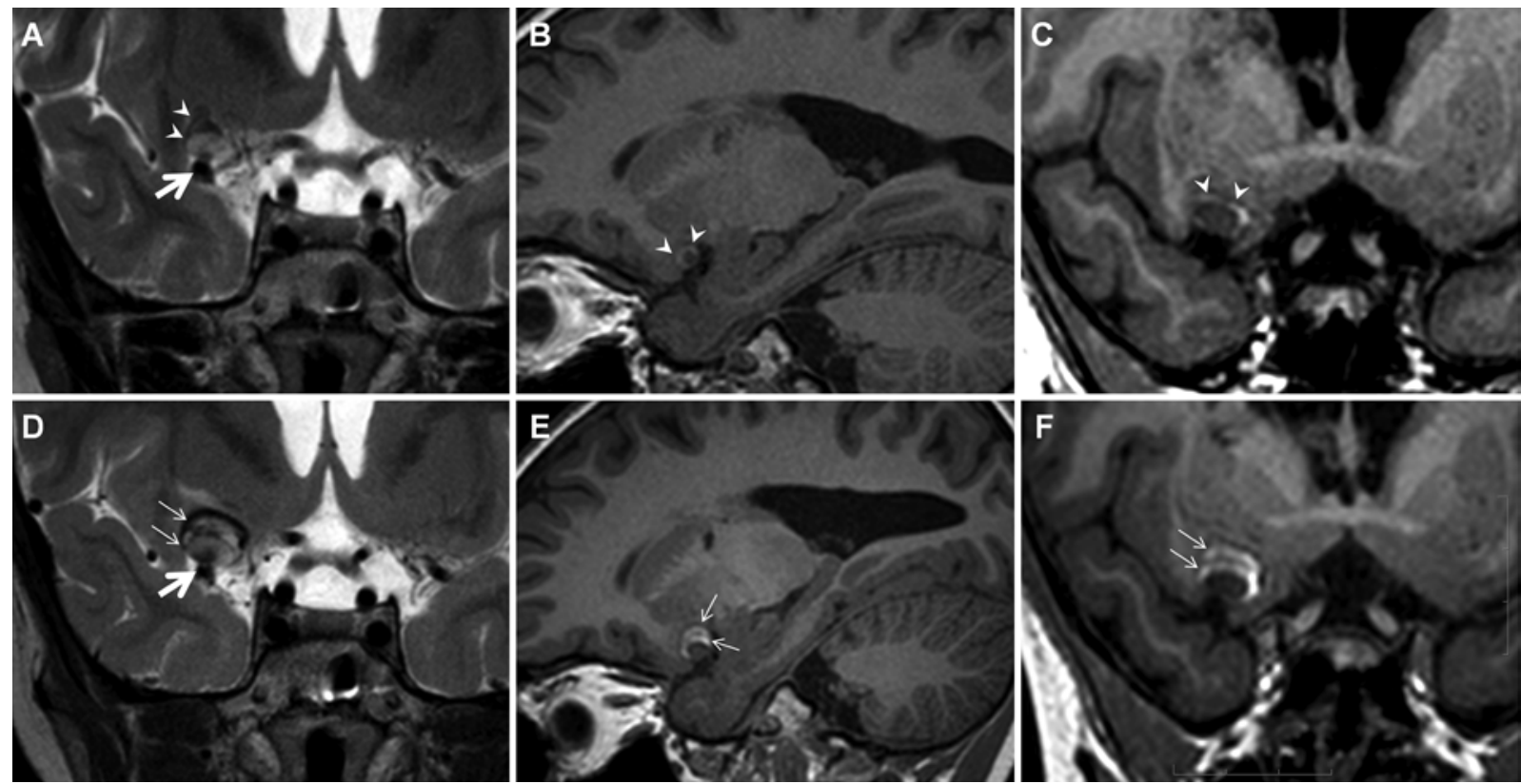

FIG. 3. Detailed views of the dissecting aneurysm on follow-up images. In the first row the images were obtained 12 days after initial MRI; the second row shows images obtained 60 days after initial MRI. A and D: Coronal T2-weighted images. B and E: Sagittal T1-weighted images. C and F: Coronal T1-weighted images. The MRI sequences demonstrate stable size of the perfused part of the aneurysm as indicated by the T2 flow void (thick arrow in panels A and D). However, the wall of the aneurysm demonstrates an increase in size and a crescent-shaped area with T1 hypersignal change indicating methemoglobin apposition in an onion skin-like fashion (thin arrows in panels D-F) on the 60th day's follow-up MRI studies compared to the images of the 12th day's follow-up studies (arrowheads in panels A-C).

\section{Operation and Postoperative Course}

Given the progressive increase of the aneurysm, we then opted for surgical intervention with a superficial temporal artery (STA)-MCA bypass and trapping of the aneurysm. At 1-year clinical and imaging follow-up the patient was neurologically intact and did not have new areas of ischemia on imaging. The aneurysm had regressed completely. The bypass, however, was no longer patent and the patient's MCA territory was now irrigated via leptomeningeal collateral vessels of the anterior and posterior cerebral arteries.

\section{Discussion}

Schubiger et al. had, as early as 1987 , detected fresh blood clot around more remote thrombus in the periphery of giant PTAs, and proposed that the repeated occurrence of hemorrhage inside a mural thrombus was the cause of continued growth of giant PTAs. ${ }^{14}$ Subsequently, other authors reported imaging and pathological evidence for repeated intramural hemorrhage within the wall of giant PTAs, distant from the perfused lumen. . $^{1,5,12,13,16,17,19}$ Based on these reports, it was suggested that some PTAs may be caused by a chronic dissecting process. ${ }^{7,16,19}$ According to this hypothesis, an intimal tear of an intradural artery leads to an intramural hematoma that neither dissects through the adventitia to cause an acute subarachnoid hemorrhage nor reopens into the parent vessel to be the cause of repeated embolic events. Instead, the intramural hematoma may clot or even organize. If the intimal tear persists, repeated intramural hemorrhages (of varying ages) may recur, leading to continuous expansion of the mural part of the PTA thus formed. Gradual organization of the clot may eventually stimulate ingrowth of vasa vasorum (especially in elderly patients with atherosclerosis and therefore a higher propensity to harbor intracranial vasa vasorum), or a chronic inflammatory process may be triggered as recently described in chronic dissecting aneurysms of the aorta. ${ }^{3,20}$ Dynamic imaging evidence for an acute dissecting aneurysm evolving into a PTA has, however, been lacking up to now.

The initial manifestation in the presented pediatric patient was a perforator infarction in the territory of the lateral lenticulostriate arteries. Although causes for pediatric stroke are manifold, arterial dissection is not uncommonly considered a potential cause of vasculopathy affecting the proximal MCA and its perforators. ${ }^{2,15}$ Initial imaging in our patient demonstrated focal narrowing of the MCA at the presumed level of the perforator origins. In addition, distal to the narrowing an irregular aneurysm was found, further underlining the dissecting nature of this disease. ${ }^{10}$ Subsequent MRI studies revealed repeated episodes of intramural hematoma, gradually evolving into a lesion with imaging characteristics as previously described in PTAs, including $\mathrm{T} 1$ hyperintensity indicating methemoglobin and an onion skin-like layering of hematomas of varying ages. ${ }^{1,6,17}$ Growth of the aneurysm was revealed to occur due to the repeated hemorrhages that were distant from the perfused lumen, with stable imaging characteristics of the focally dilated lumen.

Serial MRIs provided evidence that some PTAs can evolve from acute dissecting processes, indicating that these aneurysms are a disease of the wall rather than of the lumen. ${ }^{9}$ Recognition of the nature of this disease has direct 
implications for treatment because endoluminal treatment methods such as coil insertion would not be effective; the extraluminal disease may still lead to continued growth of the aneurysm. ${ }^{4}$ Giant PTAs represent a therapeutic challenge and, ideally, complete surgical removal or trapping of the aneurysm (which may necessitate bypass surgery) was deemed to be the treatment of choice. ${ }^{8}$

Because some dissecting aneurysms initially may be asymptomatic, ${ }^{8,16}$ it may be hypothesized that some patients with chronic dissecting aneurysms may only present with symptoms caused by mass effect after their dissecting aneurysm has evolved into a PTA. Given this presumed pathomechanism, it may be more accurate to refer to these lesions as aneurysms with intramural hematoma instead of "partially thrombosed" aneurysms. It is important to keep in mind that this is only one of potentially many pathomechanisms of these lesions, and that in certain cases not only mural but also intraluminal thrombus may be present.

Nevertheless, this case report may lend support to the hypothesis that some aneurysms with an onion skin-like appearance on MRI sequences are related to a group of diseases that are primarily located in the wall rather than the lumen, which may impact the selection of therapeutic management and potentially patient outcomes.

\section{References}

1. Atlas SW, Grossman RI, Goldberg HI, Hackney DB, Bilaniuk LT, Zimmerman RA: Partially thrombosed giant intracranial aneurysms: correlation of MR and pathologic findings. Radiology 162:111-114, 1987

2. Braun KP, Bulder MM, Chabrier S, Kirkham FJ, Uiterwaal CS, Tardieu M, et al: The course and outcome of unilateral intracranial arteriopathy in 79 children with ischaemic stroke. Brain 132:544-557, 2009

3. Connolly ES Jr, Huang J, Goldman JE, Holtzman RN: Immunohistochemical detection of intracranial vasa vasorum: a human autopsy study. Neurosurgery 38:789-793, 1996

4. Dehdashti AR, Thines L, Willinsky RA, Tymianski M: Symptomatic enlargement of an occluded giant carotidoophthalmic aneurysm after endovascular treatment: the vasa vasorum theory. Acta Neurochir (Wien) 151:1153-1158, 2009

5. Kaneko T, Nomura M, Yamashima T, Suzuki M, Yamashita $\mathrm{J}$ : Serial neuroimaging of a growing thrombosed giant aneurysm of the distal anterior cerebral artery-case report. Neurol Med Chir (Tokyo) 41:33-36, 2001

6. Krings T, Alvarez H, Reinacher P, Ozanne A, Baccin CE, Gandolfo C, et al: Growth and rupture mechanism of partially thrombosed aneurysms. Interv Neuroradiol 13:117-126, 2007

7. Krings T, Choi IS: The many faces of intracranial arterial dissections. Interv Neuroradiol 16:151-160, 2010

8. Krings T, Geibprasert S, terBrugge KG: Pathomechanisms and treatment of pediatric aneurysms. Childs Nerv Syst 26:1309-1318, 2010

9. Krings T, Lasjaunias PL, Geibprasert S, Pereira V, Hans FJ:
The aneurysmal wall. The key to a subclassification of intracranial arterial aneurysm vasculopathies? Interv Neuroradiol 14 Suppl 1:39-47, 2008

10. Mizutani T, Miki Y, Kojima H, Suzuki H: Proposed classification of nonatherosclerotic cerebral fusiform and dissecting aneurysms. Neurosurgery 45:253-260, 1999

11. Molyneux A, Kerr R, Stratton I, Sandercock P, Clarke M, Shrimpton J, et al: International Subarachnoid Aneurysm Trial (ISAT) of neurosurgical clipping versus endovascular coiling in 2143 patients with ruptured intracranial aneurysms: a randomised trial. Lancet 360:1267-1274, 2002

12. Nagahiro S, Takada A, Goto S, Kai Y, Ushio Y: Thrombosed growing giant aneurysms of the vertebral artery: growth mechanism and management. J Neurosurg 82:796-801, 1995

13. Nakatomi H, Segawa H, Kurata A, Shiokawa Y, Nagata K, Kamiyama H, et al: Clinicopathological study of intracranial fusiform and dolichoectatic aneurysms: insight on the mechanism of growth. Stroke 31:896-900, 2000

14. Schubiger O, Valavanis A, Wichmann W: Growth-mechanism of giant intracranial aneurysms; demonstration by $\mathrm{CT}$ and MR imaging. Neuroradiology 29:266-271, 1987

15. Sébire G, Fullerton H, Riou E, deVeber G: Toward the definition of cerebral arteriopathies of childhood. Curr Opin Pediatr 16:617-622, 2004

16. Songsaeng D, Srivatanakul K, Krings T, Geibprasert S, Ozanne A, Lasjaunias P: Symptomatic spontaneous vertebrobasilar dissections in children: review of 29 consecutive cases. J Neurosurg Pediatr 6:233-243, 2010

17. Teng MM, Nasir Qadri SM, Luo CB, Lirng JF, Chen SS, Chang CY: MR imaging of giant intracranial aneurysm. J Clin Neurosci 10:460-464, 2003

18. Wiebers DO, Whisnant JP, Huston J III, Meissner I, Brown RD Jr, Piepgras DG, et al: Unruptured intracranial aneurysms: natural history, clinical outcome, and risks of surgical and endovascular treatment. Lancet 362:103-110, 2003

19. Yasui T, Sakamoto H, Kishi H, Komiyama M, Iwai Y, Yamanaka K, et al: Rupture mechanism of a thrombosed slowgrowing giant aneurysm of the vertebral artery-case report. Neurol Med Chir (Tokyo) 38:860-864, 1998

20. Zhao L, Moos MP, Gräbner R, Pédrono F, Fan J, Kaiser B, et al: The 5-lipoxygenase pathway promotes pathogenesis of hyperlipidemia-dependent aortic aneurysm. Nat Med 10:966-973, 2004

\section{Author Contributions}

Conception and design: Krings. Acquisition of data: Krings, Yau, deVeber, Dirks, Armstrong. Analysis and interpretation of data: all authors. Drafting the article: Chen. Critically revising the article: Krings, Yau, deVeber, Dirks, Armstrong. Reviewed submitted version of manuscript: all authors. Approved the final version of the manuscript on behalf of all authors: Krings. Study supervision: Krings.

\section{Correspondence}

Timo Krings, University of Toronto, Toronto Western Hospital, UHN Divisions of Neuroradiology and Neurosurgery, 399 Bathurst St., 3MCL-429 Toronto, ON M5T 2S8, Canada. email: timo.krings@uhn.ca. 